\title{
Resolução CONAMA 357/2005: análise espacial e temporal de não conformidades em rios e reservatórios do estado de São Paulo de acordo com seus enquadramentos (2005-2009)
}

CONAMA Framework Resolution 357/2005: spatial and temporal analysis of water quality legal compliances in rivers and reservoirs from São Paulo State, Brazil (2005-2009)

\section{Davi Gasparini Fernandes Cunha}

Doutor em Ciências (Hidráulica e Saneamento) pela Universidade de São Paulo (USP). Pós-Doutorando do Departamento de Hidráulica e Saneamento da Escola de Engenharia de São Carlos, Universidade de São Paulo (SHS/EESC/USP) - São Carlos (SP), Brasil.

\section{Maria do Carmo Calijuri}

Doutora em Engenharia Hidráulica e Saneamento pela Universidade de São Paulo (USP). Professora Titular do SHS/EESC/USP - São Carlos (SP), Brasil.

\section{Marta Condé Lamparelli}

Doutora em Ecologia pela USP. Gerente da Divisão de Análises Hidrobiológicas da Companhia Ambiental do Estado de São Paulo (CETESB) - São Paulo (SP), Brasil.

Nelson Menegon Jr.

Mestre em Engenharia Hidráulica pela USP. Gerente da Divisão de Qualidade das Águas e do Solo da (CETESB) - São Paulo (SP), Brasil.

\section{Resumo}

A Resolução CONAMA 357/2005 estabeleceu condições de qualidade para o enquadramento dos sistemas aquáticos no Brasil. Essa pesquisa avaliou os níveis de não conformidade entre a qualidade de rios e reservatórios do estado de São Paulo e o seu enquadramento para algumas variáveis. Foram analisados dados $(n=43.897)$ de fósforo total, oxigênio dissolvido, nitrato, turbidez e Demanda Bioquímica de Oxigênio de 360 pontos amostrais nas 22 UGRHIs (Unidades de Gerenciamento de Recursos Hídricos) de SP. A situação se apresentou mais crítica para o fósforo total e o oxigênio dissolvido, sobretudo nas UGRHIs industriais. Entre 2005 e 2009, não houve melhoria significativa em relação aos resultados desconformes para algumas variáveis. Há necessidade de investimentos no tratamento de esgotos em nível terciário e de medidas para atenuar a geração de cargas difusas.

Palavras-chave: enquadramento dos corpos de água; estado de São Paulo; qualidade da água; Resolução CONAMA 357/2005; usos múltiplos.

\section{Abstract}

The CONAMA Framework Resolution 357/2005 fixed the conditions for establishing water quality categories in Brazilian aquatic systems. We assessed the levels of non-conformity among the water quality in rivers and reservoirs from São Paulo State (SP, Brazil) and their framework. We analyzed data ( $\mathrm{n}=43,897)$ from total phosphorus, dissolved oxygen, nitrate, turbidity and Biochemical Oxygen Demand of 360 sampling sites within the 22 UGRHI (Water Resources Management Units) in SP from 2005 to 2009. The situation was unsatisfactory for total phosphorus and dissolved oxygen, mainly in the industrial UGRHI. There was no significant water quality improvement in relation to some of the studied variables within these five years, which indicates the need for directing investments on tertiary domestic wastewater treatment and mitigation of non-point pollution loads.

Keywords: CONAMA Framework Resolution 357/2005; multiple uses; São Paulo State; water bodies framework; water quality. 


\section{Introdução}

O gerenciamento integrado dos recursos hídricos, por sua relevância, desperta significativo interesse em diferentes países. O objetivo de viabilizar a utilização da água para as atividades humanas associada à manutenção dos serviços ambientais e da biodiversidade dos ecossistemas aquáticos representa, sem dúvida, um passo fundamental em direção à sustentabilidade. Na Europa, esforços nesse sentido resultaram na Water Framework Directive, que visa a atingir, por meio de metas progressivas, um "estado ecológico satisfatório" dos cursos de água europeus até o ano de 2015 (Achleitner et al., 2005; Carone et al., 2009). Uma das ferramentas dessa diretiva é a separação dos rios em diferentes tipos, de acordo com um sistema de classificação que considera, por exemplo, a eco-região em que o sistema aquático se localiza, a altitude, o tamanho da bacia hidrográfica, além de atributos geológicos (CARBALLO et al., 2009). Iniciativas individuais em outros países europeus e de outros continentes também merecem destaque (e.g. CHEN; LIU; LEU, 2006; SILVA \& RIBEIRO, 2007; BONGARTZ et al., 2007; IORISA; HUNTERB; WALKERC, 2008). O esquema normalmente envolvido no processo de gestão dos recursos hídricos considera elementos como capacidade suporte do corpo de água, padrões de qualidade, emissão de poluentes, disponibilidade tecnológica e aspectos sociais.

Especificamente no Brasil, o Conselho Nacional de Meio Ambiente, por meio da Resolução CONAMA 357, de 17 de março de 2005 (BRASIL, 2005), estabeleceu condições de qualidade para o enquadramento dos corpos hídricos em território nacional, de acordo com os seus usos preponderantes, e para o lançamento de efluentes. Essa resolução, como instrumento jurídico, fixou limites superiores ou inferiores (alguns deles são apresentados na Tabela 1) para diversas variáveis em sistemas de água doce, salobra e salina. Desde o início de sua vigência, o documento tem sido analisado tecnicamente pela comunidade científica (e.g. REIS \& MENDONÇA, 2009) e se tornou referência inclusive para pesquisas acadêmicas. Tais pesquisas comumente promovem uma comparação entre os resultados obtidos em determinado ambiente aquático e os respectivos limites associados ao seu enquadramento.

Tabela 1 - Limites superiores ou inferiores, dependendo do caso, para algumas variáveis em ambientes aquáticos de água doce de Classes 1 , 2, 3 ou 4 (Resolução CONAMA 357/2005).

\begin{tabular}{lccccc} 
Variável & $\begin{array}{l}\text { Natureza } \\
\text { do limite }\end{array}$ & Classe 1 & Classe 2 & Classe 3 & Classe 4 \\
\hline PT $\left(\mathrm{mg} . \mathrm{L}^{-1}\right)$ & Superior & $\begin{array}{c}0,02- \\
0,10^{\mathrm{a}}\end{array}$ & $\begin{array}{c}0,03- \\
0,10^{\mathrm{a}}\end{array}$ & $\begin{array}{c}0,05- \\
0,15^{\mathrm{a}}\end{array}$ & $\mathrm{b}$ \\
\hline $\mathrm{OD}\left(\mathrm{mg} \cdot \mathrm{L}^{-1}\right)$ & Inferior & 6 & 5 & 4 & 2 \\
\hline $\mathrm{NO}_{3}\left(\mathrm{mg} \cdot \mathrm{L}^{-1}\right)$ & Superior & 10,0 & 10,0 & 10,0 & $\mathrm{~b}$ \\
\hline Turb $(\mathrm{UT})$ & Superior & 40 & 100 & 100 & $\mathrm{~b}$ \\
\hline $\begin{array}{l}\mathrm{DBO} \\
\left(\mathrm{mg} \cdot \mathrm{L}^{-1}\right)\end{array}$ & Superior & 3 & 5 & 10 & $\mathrm{~b}$ \\
\hline
\end{tabular}

PT: fósforo total; $\mathrm{OD}$ : oxigênio dissolvido; $\mathrm{NO}_{3}$ : nitrato; Turb: turbidez; $\mathrm{DBO}_{5,20}$ : Demanda Bioquímica de Oxigênio; a O limite varia para ambientes lênticos, lóticos, intermediários e

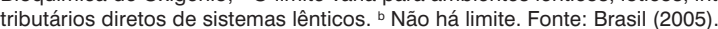

Ainda são escassos levantamentos do nível de não compatibilidade de rios e reservatórios com o seu enquadramento legal em escalas maiores de tempo e espaço. Isso permitiria uma visão menos pontual da situação desses corpos hídricos após o início da vigência da Resolução CONAMA 357/2005. Com vistas a suprir essa carência de informações mais representativas da dinâmica da qualidade da água, a presente pesquisa teve como objetivo principal a análise da heterogeneidade espacial e da evolução temporal dos conflitos que ocorreram entre os resultados do monitoramento de sistemas aquáticos no estado de São Paulo e o seus respectivos enquadramentos - com base no Decreto Estadual 10.755/1977 (SÃO PAULO, 1977).

Ao todo, 360 pontos amostrais (317 em ambientes lóticos e 43 em lênticos) compuseram a presente avaliação, o que garantiu representatividade à análise da variação temporal dos mais de 40.000 dados disponíveis, considerando dados levantados entre 2005 e 2009. O caráter espacial da investigação, por sua vez, foi atendido por meio do estudo de estações de coleta localizadas nas diferentes UGRHIs (Unidades de Gerenciamento de Recursos Hídricos) do estado de São Paulo. Dessa maneira, foi possível fazer inferências acerca dos impactos originados por áreas agrícolas e urbanas sobre a qualidade da água em comparação a regiões mais preservadas, com remanescentes florestais.

Em quaisquer estudos ambientais, é fundamental abordar essas duas escalas: a espacial e a temporal. Ao se considerarem as UGRHIs como unidades espaciais, a heterogeneidade da qualidade da água pode ser influenciada por dois fatores principais: contribuição natural da bacia hidrográfica (e.g. concentrações naturais, também conhecidas como background, afetadas, por exemplo, pelas características geológicas e pedológicas do local) e magnitude dos impactos antrópicos (e.g. consumo de água, construção de barragens, lançamento de efluentes domésticos e industriais, escoamento superficial de áreas rurais e urbanas). Do ponto de vista temporal, oscilações na qualidade da água podem refletir ações positivas de manejo na UGRHI ou, por outro lado, efeitos deletérios da intensificação de formas de uso e ocupação do solo que comprometem os ambientes aquáticos. A necessidade de conciliar aspectos quantitativos e qualitativos dos recursos hídricos nas escalas espacial e temporal e a crescente demanda de água para as atividades antrópicas, situação agravada com a degradação de rios e reservatórios, é uma tarefa complexa, pois envolve as esferas ambiental, econômica e social (CALIJURI; CUNHA; POVINELLI, 2010).

\section{Metodologia}

\section{Área de estudo}

O estado de São Paulo se localiza na Região Sudeste do Brasil e ocupa uma área de aproximadamente $248 \mathrm{mil} \mathrm{km}^{2}$, na qual 
se distribuem 645 municípios com população total superior a 42 milhões de habitantes (SEADE, 2010). O território foi dividido em 22 UGRHIs (Figura 1, Tabela 2) para facilitar o gerenciamento dos recursos hídricos e permitir uma classificação mais precisa dessas unidades de acordo com a forma de uso e ocupação do solo predominante. Quatro unidades foram classificadas como aptas a conservação ambiental, 10 como industrializadas ou com industrialização em curso (totalizam mais de $80 \%$ da população do estado) e oito como agropecuárias. As menores densidades populacionais são verificadas nas UGHRIs com aptidão para conservação e para agropecuária, com 34 e 45 hab. km², respectivamente, embora a UGRHI 3, localizada no litoral norte do estado, apresente densidade elevada, 140 hab. $\mathrm{km}^{-2}$. A máxima densidade é de 3.342 hab. $\mathrm{km}^{-2}$ na UGRHI 6 (Alto Tietê), de vocação industrial.

Até mesmo as unidades destinadas à conservação ambiental são afetadas pelas atividades humanas. Há relatos da presença de pesticidas em cursos de água da bacia hidrográfica do rio Ribeira de Iguape, UGRHI 11 (MARQUES et al., 2007) e do impacto negativo de efluentes industriais nessa região (Calijuri et al., 2008; CUNHA \& CALIJURI, 2010). Situações críticas de escassez de água já ocorrem em algumas UGRHIs, como resultado da elevada densidade demográfica aliada à relativa baixa disponibilidade hídrica e à degradação dos mananciais, como, por exemplo, na UGRHI 5 (MARCON, 2005; ROMÁN; FOLEGATTI; GONZÁLEZ, 2010).

Embora cada UGRHI possua particularidades que gerem reflexos negativos na qualidade da água, partiu-se da hipótese de que o lançamento de esgotos domésticos não tratados nos corpos hídricos é uma das principais causas do aumento do nível de trofia de rios e reservatórios. Para isso, foram selecionadas algumas variáveis monitoradas regularmente pela CETESB (Companhia Ambiental do Estado de São Paulo) no período de 2005 a 2009, tais como fósforo total (PT), oxigênio dissolvido (OD), nitrato $\left(\mathrm{NO}_{3}\right)$, turbidez (Turb) e Demanda Bioquímica de Oxigênio

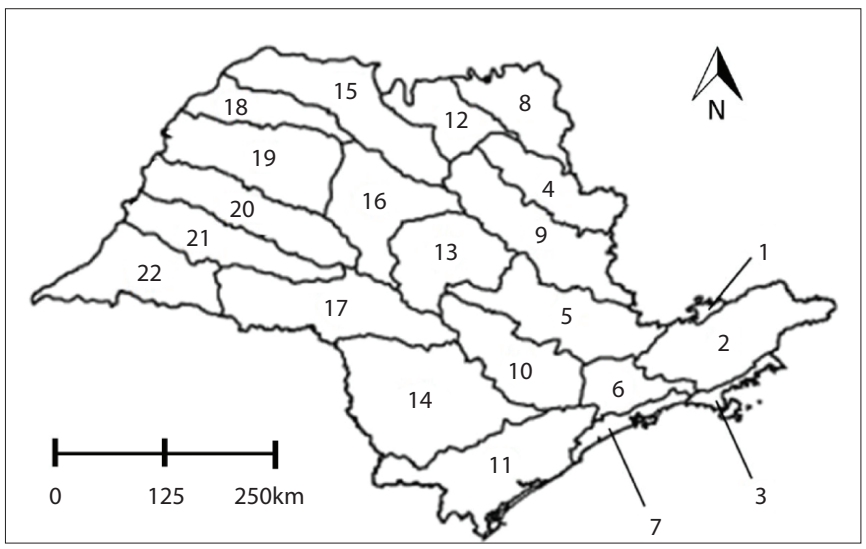

Figura 1 - Mapa do estado de São Paulo segundo a divisão do território em UGRHIs (Unidades de Gerenciamento de Recursos Hídricos), numeradas de 1 a 22.
$\left(\mathrm{DBO}_{5,20}\right)$. Essas variáveis foram escolhidas porque estão diretamente relacionadas à eutrofização de rios e reservatórios e por refletirem os impactos das formas de uso do solo, bem como das intervenções humanas sobre os corpos de água.

\section{Análise dos dados}

Os resultados disponíveis de qualidade da água (CETESB, 2005, 2006, 2007, 2008, 2009) foram analisados sob os aspectos espacial e temporal. Primeiramente, uma avaliação dos dados foi realizada por meio do cálculo de concentrações médias, valores

Tabela 2 - Classificação das 22 UGRHIs do estado de São Paulo, segundo o uso preponderante do solo (conservação, industrial, em industrialização ou agropecuária) e respectivos dados de área territorial, população e densidade populacional.

\begin{tabular}{|c|c|c|c|c|c|}
\hline \multicolumn{3}{|l|}{ UGRHI } & \multirow{2}{*}{$\begin{array}{l}\text { Área } \\
\left(\mathrm{km}^{2}\right)\end{array}$} & \multirow{2}{*}{$\begin{array}{c}\begin{array}{c}\text { Popula- } \\
\text { ção }\end{array} \\
\text { (10 hab) }\end{array}$} & \multirow{2}{*}{$\begin{array}{c}\text { Densidade } \\
\text { populacio- } \\
\text { nal }\end{array}$} \\
\hline $\begin{array}{l}\text { Classificação/ } \\
\text { aptidão }\end{array}$ & $\begin{array}{l}\text { Núme- } \\
\text { ro }\end{array}$ & Nome & & & \\
\hline \multirow{5}{*}{ Conservação } & 1 & Mantiqueira & 675 & 64 & 95 \\
\hline & 3 & Litoral Norte & 1.948 & 272 & 140 \\
\hline & 11 & $\begin{array}{l}\text { Ribeira de } \\
\text { Iguape }\end{array}$ & 17.068 & 380 & 22 \\
\hline & 14 & $\begin{array}{l}\text { Alto Paranapa- } \\
\text { nema }\end{array}$ & 22.689 & 736 & 33 \\
\hline & & subtotal & 42.380 & 1.452 & 34 \\
\hline \multirow{6}{*}{ Industrial } & 2 & Paraíba do Sul & 14.444 & 1.992 & 138 \\
\hline & 5 & $\begin{array}{c}\text { Piracicaba, } \\
\text { Capivari e } \\
\text { Jundiaí }\end{array}$ & 14.178 & 5.038 & 355 \\
\hline & 6 & Alto Tietê & 5.868 & 19.611 & 3.342 \\
\hline & 7 & $\begin{array}{l}\text { Baixada } \\
\text { Santista }\end{array}$ & 2.818 & 1.668 & 592 \\
\hline & 10 & $\begin{array}{l}\text { Sorocaba e } \\
\text { Médio Tietê }\end{array}$ & 11.829 & 1.821 & 154 \\
\hline & & subtotal & 49.137 & 30.130 & 613 \\
\hline \multirow{6}{*}{$\begin{array}{l}\text { Em } \\
\text { industrializa- } \\
\text { ção }\end{array}$} & 4 & Pardo & 8.993 & 1,068 & 119 \\
\hline & 8 & $\begin{array}{l}\text { Sapucaí- } \\
\text {-Grande }\end{array}$ & 9.125 & 689 & 76 \\
\hline & 9 & Mogi-Guaçu & 15.004 & 1.450 & 97 \\
\hline & 12 & $\begin{array}{l}\text { Baixo Pardo- } \\
\text { Grande }\end{array}$ & 7.239 & 332 & 46 \\
\hline & 13 & Tietê-Jacaré & 11.779 & 1,505 & 128 \\
\hline & & subtotal & 52.140 & 5.043 & 97 \\
\hline \multirow{9}{*}{ Agropecuária } & 15 & Turvo-Grande & 15.925 & 1,247 & 78 \\
\hline & 16 & Tietê-Batalha & 13.149 & 518 & 39 \\
\hline & 17 & $\begin{array}{l}\text { Médio Parana- } \\
\text { panema }\end{array}$ & 16.749 & 681 & 41 \\
\hline & 18 & $\begin{array}{l}\text { São José dos } \\
\text { Dourados }\end{array}$ & 6.783 & 228 & 34 \\
\hline & 19 & Baixo Tietê & 15.588 & 759 & 49 \\
\hline & 20 & Aguapeí & 13.196 & 366 & 28 \\
\hline & 21 & Peixe & 10.769 & 466 & 43 \\
\hline & 22 & $\begin{array}{l}\text { Pontal do Para- } \\
\text { napanema }\end{array}$ & 12.395 & 483 & 39 \\
\hline & & subtotal & 104.554 & 4.748 & 45 \\
\hline Todas & & TOTAL & 248.209 & 41.374 & 167 \\
\hline
\end{tabular}

Fonte: CETESB (2009) 
mínimos e máximos. Foram construídas curvas de frequência acumulada (i.e. curvas de permanência) para cada uma das variáveis, conforme sugerido por Cunha e Calijuri (2010). Em seguida, os dados foram separados por UGRHI para a avaliação do grau de não conformidade dos sistemas aquáticos com o seu enquadramento legal em cada unidade de gerenciamento. Nessa etapa, foi empregada Análise de Variância (ANOVA) para verificar se a componente espacial foi de fato relevante para a variação dos dados, com nível de confiança de 95\% ( $\left.p^{*}<0,05\right)$, que indica se os dados são estatisticamente semelhantes ou distintos. Por fim, todos os dados foram considerados, sem a distinção por tipo de UGRHIs (conservação, agropecuárias, em industrialização e industriais), mas separadamente para os anos de 2005 a 2009. Assim, pôde-se observar a evolução dos casos de conflito com o enquadramento de rios e reservatórios ao longo do tempo. Para essa análise, foram considerados apenas os pontos amostrais para os quais havia resultados disponíveis para os cinco anos avaliados (184 pontos em rios e 22 em reservatórios). Análise de Variância também foi empregada para verificar a existência de diferença estatística entre os resultados do monitoramento no período analisado.

\section{Resultados e discussão}

\section{Caracterização geral dos dados}

Foram analisados 43.897 dados para as variáveis estudadas entre 2005 e 2009, referentes a 317 pontos de amostragem em rios e 43 em reservatórios do estado de São Paulo. Para cada variável, o número de dados disponíveis esteve próximo a 7.000 para rios e 1.000 para reservatórios. A análise dos valores ou das concentrações médias (Tabela 3) e das curvas de permanência das variáveis estudadas

Tabela 3 - Valores e concentrações médios (as), mínimos (as) e máximos (as) para algumas variáveis da água de rios e reservatórios do estado de São Paulo e respectivos números de dados disponíveis.

\begin{tabular}{|c|c|c|c|c|c|}
\hline \multirow{2}{*}{ Variável } & \multicolumn{2}{|r|}{ Rios } & \multicolumn{2}{|c|}{ Reservatórios } & \multirow{2}{*}{$\begin{array}{c}\text { Total por } \\
\text { variável } \\
\text { n }\end{array}$} \\
\hline & $n$ & $\begin{array}{c}\text { Méd } \\
\text { (Min-Máx) }\end{array}$ & $\mathrm{n}$ & $\begin{array}{c}\text { Méd } \\
\text { (Min-Máx) }\end{array}$ & \\
\hline PT (mg. L-1) $^{-1}$ & 7.110 & $\begin{array}{c}0,43 \\
(<0,01-65,0)\end{array}$ & 1.200 & $\begin{array}{c}0,20 \\
(<0,01-9,5)\end{array}$ & 8.310 \\
\hline $\mathrm{OD}\left(\mathrm{mg} \cdot \mathrm{L}^{-1}\right)$ & 7.966 & $\begin{array}{c}5,0 \\
(<0,1-15,0)\end{array}$ & 1.181 & $\begin{array}{c}6,4 \\
(<0,1-16,4)\end{array}$ & 9.147 \\
\hline $\mathrm{NO}_{3}\left(\mathrm{mg} \cdot \mathrm{L}^{-1}\right)$ & 7.057 & $\begin{array}{c}0,8 \\
(<0,01-55,0)\end{array}$ & 1.194 & $\begin{array}{c}0,7 \\
(0,01-84,0)\end{array}$ & 8.251 \\
\hline Turb (UT) & 7.891 & $\begin{array}{c}50 \\
(<1-4.000)\end{array}$ & 1.165 & $\begin{array}{c}18 \\
(<1-4.000)\end{array}$ & 9.056 \\
\hline \multirow[t]{2}{*}{$\begin{array}{l}\mathrm{DBO}_{5,20} \\
\left(\mathrm{mg}_{.-1} \mathrm{~L}^{-1}\right)\end{array}$} & 7.957 & $\begin{array}{c}9 \\
(<1-427)\end{array}$ & 1.176 & $\begin{array}{c}6 \\
(<1-129)\end{array}$ & 9.133 \\
\hline & & & & & $\begin{array}{l}\text { Total: } \\
43.897\end{array}$ \\
\hline
\end{tabular}

Méd: concentração ou valor médio (a); Min: concentração ou valor mínimo (a); Máx: concentração ou valor máximo (a)
(Figura 2) sugeriu que os rios apresentaram pior qualidade da água em relação aos reservatórios no período de 2005 a 2009. Isso pode estar vinculado ao papel mais significativo de mecanismos de depuração da água nos reservatórios, sobretudo pelos processos de sedimentação e de assimilação de nutrientes pelas comunidades biológicas para produção primária, o que pode levar à eutrofização dos corpos de água e, eventualmente, a danos ambientais e econômicos (DODDS et al., 2009). As medianas foram, respectivamente para rios e reservatórios, de 0,12 e 0,04 mg. $\mathrm{L}^{-1}(\mathrm{PT}), 5,5$ e 7,0 mg. $\mathrm{L}^{-1}$ (OD), 0,35 e 0,20 mg. L-1 $\left(\mathrm{NO}_{3}\right), 20$ e $6 \mathrm{UT}$ (Turb) e 3 e $3 \mathrm{mg} \cdot \mathrm{L}^{-1}\left(\mathrm{DBO}_{5,20}\right)$.

O estudo de Lamparelli (2004), que avaliou o grau de trofia de corpos de água do estado de São Paulo entre os anos de 1996 e 2001, relatou concentrações médias de fósforo total iguais a 0,19 e 0,08 mg. $\mathrm{L}^{-1}$ em 35 pontos amostrais em rios e 34 em reservatórios, respectivamente $(n=1.419)$, excetuando-se os sistemas aquáticos Classe 4. Analogamente, excluindo-se da análise os pontos Classe 4 , as concentrações médias de fósforo obtidas pela presente pesquisa, que se refere a um período posterior (2005 a 2009), foram de 0,31 e 0,13 mg. $\mathrm{L}^{-1}$ em rios e reservatórios, respectivamente.

Entre os anos de 2005 e 2009, as maiores porcentagens de não conformidade com o enquadramento legal foram observadas para as variáveis fósforo total e oxigênio dissolvido, não apenas para os rios (48 e 39\%, respectivamente) mas também para os reservatórios (61 e 27\%). Para a $\mathrm{DBO}_{5,20}$, as porcentagens foram de $21 \%$ para sistemas lóticos e lênticos (Tabela 4). Em estudo similar realizado no rio Salgado (CE), Sabiá (2008) relatou cinco parâmetros em desacordo com os padrões de qualidade (OD, DBO, coliformes termotolerantes, nitrogênio amoniacal e PT) e propôs o reenquadramento do corpo hídrico. Caso semelhante foi reportado para o rio Corumbataí (SP) por Belondi (2003). De acordo com o pesquisador, a análise das projeções futuras com base nas demandas de água e na geração de cargas poluidoras indicou a dificuldade de manter um trecho do corpo hídrico como Classe 2 e a sugestão de reenquadrá-lo para a Classe 3, após a consulta aos diferentes usuários.

Os resultados sugeriram que os rios têm contribuído para o aporte de matéria orgânica aos reservatórios, o que foi ressaltado por Martinelli et al. (2002), que realizaram um levantamento das cargas orgânicas lançadas nos rios do estado de São Paulo. As cargas remanescentes, não removidas por sistemas de tratamento de efluentes, podem contribuir para a ampliação da não conformidade de PT nos reservatórios, além das cargas decorrentes do lançamento de esgotos domésticos sem tratamento, também reconhecidas como um dos principais fatores de degradação da qualidade das águas, como verificado para o Ribeirão do Meio, São Paulo (SARDINHA et al., 2008). Além disso, a entrada de formas fosfatadas nos reservatórios é especialmente problemática considerando que esses ambientes estão mais sujeitos à eutrofização. Justamente por essa razão, os limites estabelecidos pelo enquadramento em relação ao fósforo são mais restritivos para os reservatórios em comparação aos rios. Já existem 

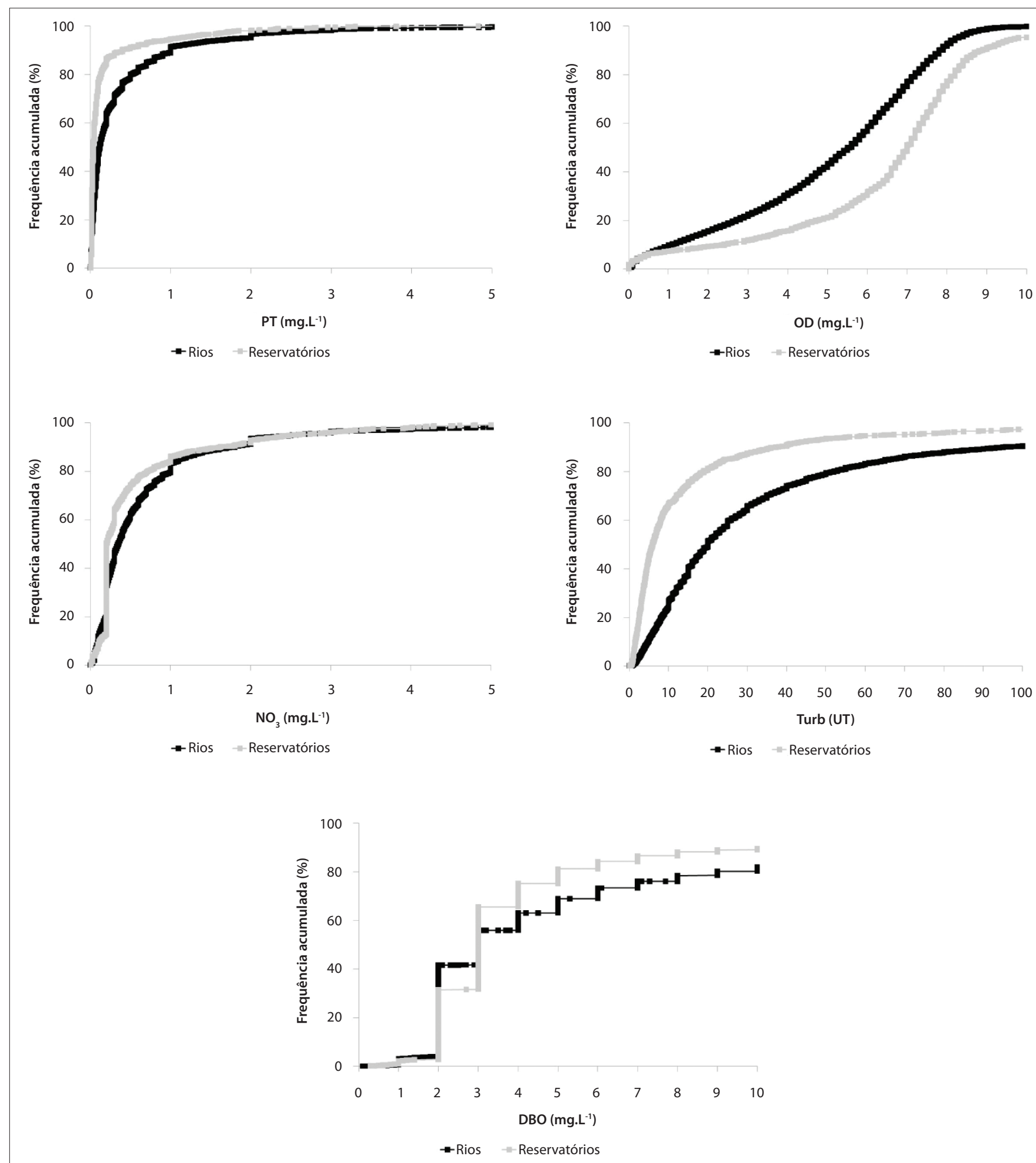

Figura 2 - Curvas de frequência acumulada (\%) dos dados das variáveis da água estudadas em rios e reservatórios do estado de São Paulo de 2005 a 2009: PT (fósforo total), OD (oxigênio dissolvido), $\mathrm{NO}_{3}$ (nitrato), Turb (turbidez) e DBO ${ }_{5,20}$ (Demanda Bioquímica de Oxigênio).

diversos relatos de reservatórios eutrofizados no estado de São Paulo (e.g. HENRY; CARMO; BICUDO, 2004; MOSCHINI-CARLOS et al., 2009). Para as variáveis nitrato e turbidez, a situação se mostrou menos crítica, uma vez que as porcentagens de não conformidade não ultrapassaram $10 \%$ em nenhum caso.

\section{Análise espacial das não conformidades com 0 enquadramento}

Em relação a todas as variáveis, os resultados desconformes foram mais frequentes nas UGRHIs industrializadas e em industrialização, 
seguidas pelas UGRHIs com vocação para agropecuária e conservação (Tabela 5). A Análise de Variância também confirmou que a qualidade da água de rios e reservatórios do estado de São Paulo variou significativamente de acordo com a vocação das UGRHIs ( $\left.p^{*}<0,05\right)$ e, consequentemente, de acordo com as formas preponderantes de uso e ocupação do solo. No caso do PT e do OD, por exemplo, as porcentagens de não conformidade nas UGRHIs industriais foram de 63 e $44 \%$, respectivamente.

No caso das UGRHIs em industrialização, algumas pesquisas comprovaram que a remoção de vegetação tem afetado a qualidade da água em relação a variáveis como cor, turbidez, alcalinidade e nitrogênio total (e.g. DONADIO; GALBIATTI; DE PAULA, 2005). As UGRHIs com vocação para agropecuária apresentam problemas ambientais vinculados ao desmatamento para instalação de propriedades rurais e à aceleração dos processos erosivos com consequente perda de solo, como ocorre na unidade do Pontal do Paranapanema (LEAL, 2000) e de São José dos Dourados (FRANCO \& HERNANDEZ, 2009).

Pode-se verificar na Tabela 5 que nas UGRHIs industriais existe maior número de pontos de monitoramento, pois elas concentram

Tabela 4 - Porcentagens de não conformidades, em rios e reservatórios, para as variáveis: fósforo total (PT), oxigênio dissolvido (OD), nitrato ( $\mathrm{NO}_{3}$ ), turbidez (Turb) e Demanda Bioquímica de Oxigênio $\left(\mathrm{DBO}_{5,20}\right)$ com relação à Resolução CONAMA 357/2005 e respectivos números de pontos amostrais referentes a cada classe de enquadramento.

\begin{tabular}{|c|c|c|c|c|c|c|c|c|}
\hline \multirow{3}{*}{ Variável } & \multicolumn{8}{|c|}{ Não conformidade com a Resolução CONAMA 357/2005 } \\
\hline & \multicolumn{3}{|c|}{ Rios } & \multicolumn{2}{|c|}{ Reservatórios } & \multicolumn{3}{|c|}{ Total } \\
\hline & Classe 2 & Classe 3 & Classe 4 & Classe 2 & Classe 4 & Rios $^{a}$ & Reservatórios ${ }^{b}$ & Rios e Reservatórios ${ }^{c}$ \\
\hline PT (\%) & 46 & 61 & - & 61 & - & 48 & 61 & 49 \\
\hline $\mathrm{OD}(\%)$ & 35 & 49 & 53 & 21 & 95 & 39 & 27 & 38 \\
\hline $\mathrm{NO}_{3}(\%)$ & 1 & 0 & - & 1 & - & 1 & 1 & 1 \\
\hline Turb (\%) & 9 & 7 & - & 4 & - & 9 & 4 & 8 \\
\hline $\mathrm{DBO}_{5,20}(\%)$ & 19 & 34 & - & 21 & - & 21 & 21 & 21 \\
\hline Número de pontos & 248 & 25 & 44 & 40 & 3 & 317 & 43 & 360 \\
\hline
\end{tabular}

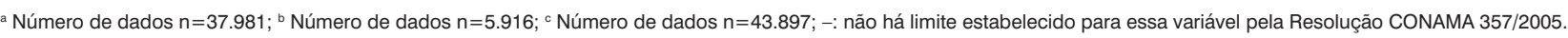

Tabela 5 - Distribuição das não conformidades (\%) de rios e reservatórios para as 22 UGRHI do estado de São Paulo, relativas à Resolução CONAMA 357/2005 para as variáveis: fósforo total (PT), oxigênio dissolvido (OD), nitrato ( $\mathrm{NO}_{3}$ ), turbidez (Turb) e Demanda Bioquímica de Oxigênio (DBO ${ }_{5,20}$ ).

\begin{tabular}{|c|c|c|c|c|c|c|}
\hline \multirow{2}{*}{ UGRHI } & \multirow{2}{*}{$\mathrm{n}$} & \multicolumn{5}{|c|}{ Não conformidade com a Resolução CONAMA 357/2005 } \\
\hline & & PT (\%) & OD (\%) & $\mathrm{NO}_{3}(\%)$ & Turb (\%) & $\mathrm{DBO}_{5,20}(\%)$ \\
\hline 1- Mantiqueira & 2 & 77 & 0 & 0 & 10 & 28 \\
\hline 3- Litoral Norte & 32 & 13 & 20 & 0 & 1 & 4 \\
\hline 11- Ribeira de Iguape & 12 & 49 & 16 & 1 & 4 & 25 \\
\hline 14- Alto Paranapanema & 12 & 24 & 9 & 0 & 12 & 5 \\
\hline UGRHI - Conservação & 58 & 25 & 16 & 0 & 4 & 9 \\
\hline 2- Paraíba do Sul & 14 & 11 & 45 & 0 & 11 & 1 \\
\hline 5- Piracicaba, Capivari e Jundiaí & 75 & 83 & 37 & 1 & 15 & 34 \\
\hline 6- Alto Tietê & 40 & 57 & 61 & 0 & 3 & 34 \\
\hline 7- Baixada Santista & 14 & 35 & 30 & 4 & 3 & 29 \\
\hline 10- Sorocaba e Médio Tietê & 30 & 75 & 46 & 1 & 9 & 40 \\
\hline UGRHI - Industrial & 173 & 63 & 44 & 1 & 11 & 31 \\
\hline 4- Pardo & 4 & 19 & 3 & 0 & 3 & 0 \\
\hline 8- Sapucaí-Grande & 13 & 22 & 2 & 0 & 10 & 3 \\
\hline 9- Mogi-Guaçu & 32 & 49 & 55 & 0 & 7 & 12 \\
\hline 12- Baixo Pardo-Grande & 3 & 27 & 7 & 0 & 0 & 0 \\
\hline 13- Tietê-Jacaré & 7 & 58 & 21 & 1 & 3 & 7 \\
\hline UGRHI - Em industrialização & 59 & 43 & 38 & 0 & 6 & 9 \\
\hline 15- Turvo-Grande & 16 & 67 & 64 & 0 & 7 & 21 \\
\hline 16- Tietê-Batalha & 22 & 16 & 15 & 1 & 2 & 2 \\
\hline 17- Médio Paranapanema & 3 & 10 & 0 & 0 & 3 & 1 \\
\hline 18- São José dos Dourados & 1 & 23 & 0 & 0 & 10 & 3 \\
\hline 19- Baixo Tietê & 11 & 18 & 21 & 0 & 1 & 4 \\
\hline 20- Aguapeí & 7 & 38 & 11 & 0 & 11 & 17 \\
\hline 21- Peixe & 4 & 54 & 11 & 1 & 30 & 5 \\
\hline 22- Pontal do Paranapanema & 6 & 25 & 5 & 2 & 8 & 23 \\
\hline UGRHI - Agropecuária & 70 & 35 & 10 & 0 & 8 & 12 \\
\hline
\end{tabular}


a maior intensidade de atividades antrópicas e a maioria da população do estado. A distribuição dos pontos nas UGRHIs de conservação e agropecuárias tem aumentado ao longo dos anos, procurando dar melhor representatividade a Rede de Monitoramento para todo o estado. No entanto, o trabalho desenvolvido por Midaglia (2009), que analisou a representatividade espacial da Rede de Monitoramento da CETESB para avaliar a evolução da qualidade da água face ao crescimento populacional, concluiu que justamente as UGRHIs industriais estariam mais sub-representadas. A não conformidade do fósforo total nas diferentes UGRHIs foi maior nas unidades que apresentaram menores porcentagens de tratamento de esgoto (Figura 3). Assim, embora provavelmente haja outros fatores intervenientes, o deficit de tratamento de águas residuárias domésticas em cada UGRHI contribuiu significativamente para o incremento das concentrações de fósforo nos respectivos corpos de água, o que levou a um aumento da porcentagem de resultados desconformes com o enquadramento.

Destacaram-se as porcentagens de não conformidade das concentrações de fósforo total com o enquadramento de corpos hídricos em algumas UGRHIs com vocação para conservação, como por exemplo, nas UGRHI 1 (77\%) e 11 (49\%). Embora esses resultados possam indicar o aumento das pressões antrópicas nessas regiões e a falta de tratamento de esgotos domésticos, a contribuição natural das bacias hidrográficas para as concentrações de fósforo também não pode ser negligenciada. A UGRHI 1, na maior parte do período estudado, foi representada por apenas um ponto de amostragem, o que inevitavelmente também interferiu na presente análise. Na UGRHI 11 (Ribeira do Iguape), predominam rochas fosfatadas, com formações geológicas referentes a jazidas de apatita e carbonatito (MOCCELLIN, 2006), o que gera efeitos

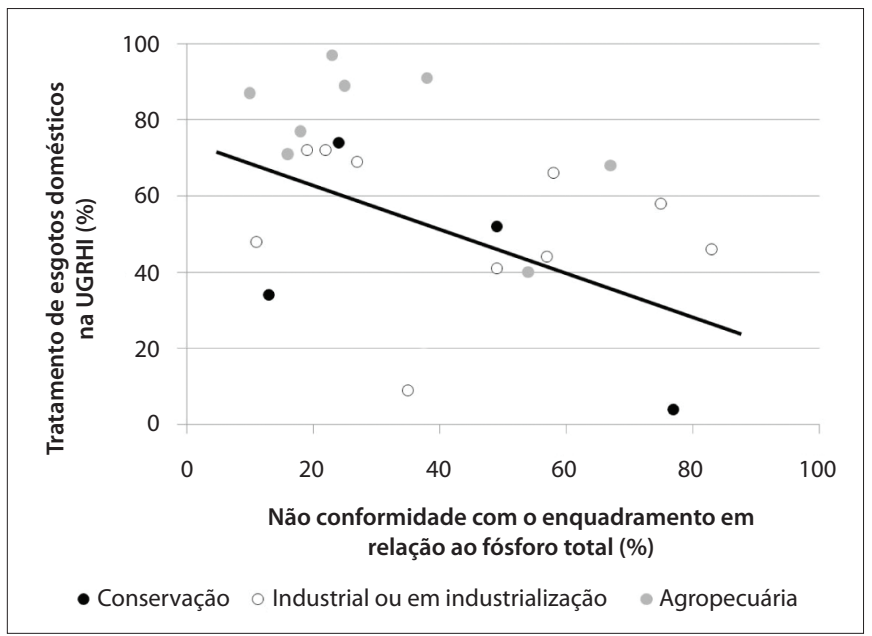

Figura 3 - Correlação entre as porcentagens de tratamento de esgoto doméstico nas UGRHI do estado de São Paulo e as porcentagens de não conformidade com o enquadramento em relação ao fósforo total. na qualidade das águas superficiais. Assim, torna-se complexa a distinção entre o incremento das concentrações de fósforo vinculado diretamente às atividades humanas e aquele que é reflexo da contribuição natural da bacia de drenagem.

$\mathrm{Na}$ tentativa de remediar essa questão, alguns pesquisadores utilizaram a abordagem por eco-regiões, principalmente em sistemas temperados (e.g. DODDS; CARNEY; ANGELO, 2006). Assim, o território de um estado é dividido em áreas de acordo com similaridades em seus atributos de clima, vegetação, geologia, entre outros. Posteriormente, são estabelecidos valores de referência da qualidade da água para cada eco-região, por meio de diversas técnicas disponíveis na literatura (DODDS \& OAKES, 2004). Esse mecanismo garante, em parte, que sejam levadas em conta especificidades naturais de cada eco-região que possam afetar as características físicas, químicas e biológicas dos cursos de água. Analogamente, para os sistemas aquáticos subtropicais de São Paulo, o levantamento dos valores de referência (background) nas diversas UGRHIs do estado subsidiaria uma avaliação mais criteriosa dos resultados não conformes com os padrões de qualidade. Isso seria possível por meio de uma análise que verificasse a distinção entre as características naturais dos corpos de água e aquelas oriundas, de fato, dos impactos de origem antrópica, conforme detalhadamente descrito por Cunha, Dodds e Calijuri (2011) e Cunha, Ogura e Calijuri (2012).

\section{Análise temporal das não conformidades com 0 enquadramento}

Entre 2005 e 2009, houve aumento de resultados desconformes para o PT (50 para 55\%) e para a turbidez (6 para 12\%); porém, houve diminuição de desconformidade para o OD (36 para 33\%).

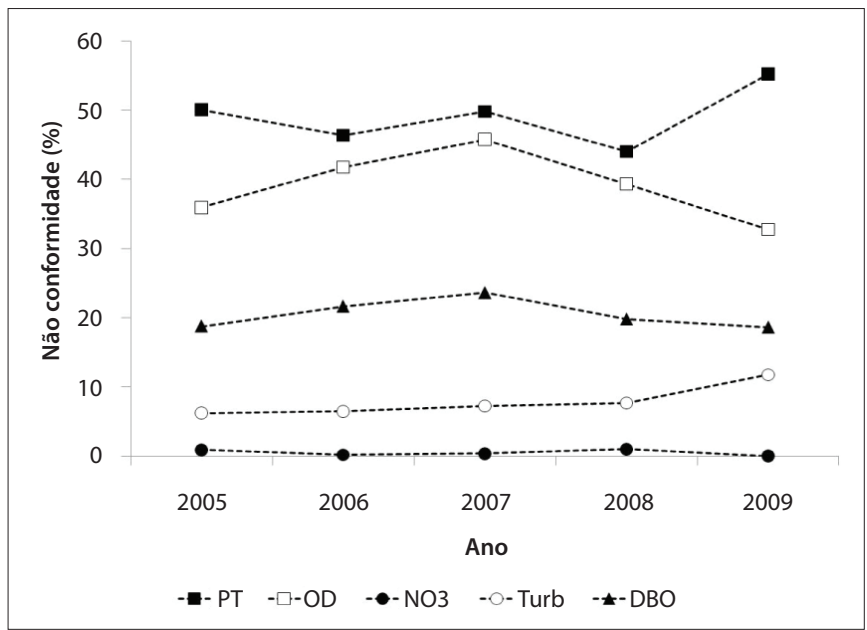

Figura 4 - Evolução temporal dos casos de não conformidades (\%) entre os sistemas aquáticos monitorados (rios e reservatórios) entre os anos de 2005 e 2009 em relação às seguintes variáveis: fósforo total (PT), oxigênio dissolvido (OD), nitrato $\left(\mathrm{NO}_{3}\right)$, turbidez (Turb) e Demanda Bioquímica de Oxigênio $\left(\mathrm{DBO}_{5,20}\right)$. 
Tabela 6 - Síntese dos resultados da Análise de Variância (ANOVA), processada para verificar a existência ou não de diferença estatisticamente significativa entre os resultados do monitoramento de rios e reservatórios entre os anos de 2005 e 2009.

\begin{tabular}{lcc} 
Variável & Rios & Reservatórios \\
$\mathrm{PT}$ & $\mathrm{p}>0,05$ & $\mathrm{p}>0,05$ \\
$\mathrm{OD}$ & $\mathrm{p}^{*}<0,05$ & $\mathrm{p}^{*}<0,05$ \\
$\mathrm{NO}_{3}$ & $\mathrm{p}^{*}<0,05$ & $\mathrm{p}^{*}<0,05$ \\
\hline Turbidez & $\mathrm{p}>0,05$ & $\mathrm{p}>0,05$ \\
$\mathrm{DBO}_{5,20}$ & $\mathrm{p}^{*}<0,05$ & $\mathrm{p}>0,05$ \\
\hline
\end{tabular}

Ao longo desses cinco anos, $\mathrm{DBO}_{5,20}$ e $\mathrm{NO}_{3}$ apresentaram-se praticamente estáveis em relação aos conflitos com o enquadramento dos rios e reservatórios (Figura 4). A Análise de Variância sugeriu que não houve variação temporal estatisticamente significativa nas concentrações de PT e valores de turbidez nos rios e nas concentrações de PT e valores de turbidez e $\mathrm{DBO}_{5,20}$ no caso dos reservatórios entre 2005 e 2009 (Tabela 6). Os resultados da ANOVA também indicaram que houve melhoria da qualidade da água ao longo do tempo em relação ao OD em rios e reservatórios, embora a porcentagem de resultados desconformes em 2009 ainda tenha sido relativamente elevada, $33 \%$ (Figura 4). No período de cinco anos investigado, caracterizado por crescimento populacional de 4,86\% (SEADE, 2005, 2009), as eventuais ações em prol do saneamento básico e da recuperação dos sistemas aquáticos no estado de São Paulo foram insuficientes para resultar em melhora significativa da qualidade da água em seus rios e reservatórios.

Com vistas à diminuição dos níveis de não compatibilidade com o enquadramento, é fundamental que haja mais investimentos em coleta e tratamento de esgotos domésticos no estado, melhoria da eficiência de Estações de Tratamento de Esgotos e em ações para atenuação das cargas difusas oriundas de episódios de chuva (CETESB, 2009). Entre 2005 e 2009, a porcentagem de tratamento de efluentes domésticos no estado de São Paulo aumentou de 39 para 49\% (CETESB, 2009). Embora tenha havido uma evolução, esse nível de tratamento, ainda insuficiente, pode ser considerado um dos maiores responsáveis pelo comprometimento dos usos múltiplos dos sistemas aquáticos em esfera estadual.

O estabelecimento de metas progressivas de melhoria da qualidade da água dos corpos de água em cada UGRHI, com cronograma e estratégias devidamente definidas, também pode contribuir para isso. Nesse sentido, deve-se resgatar a premissa de que a Resolução CONAMA 357/2005 deve servir precipuamente como ferramenta de planejamento dos recursos hídricos e não como simples documento que fixa valores característicos de cada classe de enquadramento. Esse papel é dos comitês de bacia, que podem, por meio da resolução, planejar a melhoria progressiva dos rios e reservatórios com especial atenção aos usos mais nobres da água e com vistas à sustentabilidade em longo prazo. As classes menos restritivas de qualidade da água (e.g. Classe 4) podem ser consideradas como etapas intermediárias a uma situação mais adequada, e não como objetivo final do enquadramento (PIZELLA \& SOUZA, 2007). A presente pesquisa mostrou que os ambientes de Classe 4 parecem ser considerados como irrecuperáveis ou como destino final inevitável de efluentes domésticos ou industriais, já que os resultados desconformes para o OD, por exemplo, atingiram 95\% em reservatórios assim enquadrados.

\section{Conclusões e recomendações}

A análise das não conformidades entre os resultados do monitoramento de rios e reservatórios do estado de São Paulo com a Resolução CONAMA 357/2005, em seus aspectos espaciais (i.e. nas 22 UGRHIs) e temporais (i.e. entre os anos de 2005 e 2009) permitiu que fossem estabelecidas as seguintes conclusões e recomendações:

- As variáveis fósforo total e oxigênio dissolvido foram as que apresentaram situação mais preocupante em relação aos resultados não conformes com o enquadramento de rios e reservatórios. A situação mais crítica de conflitos com o enquadramento foi verificada nas UGRHIs industrializadas e com maiores densidades populacionais, o que pode indicar maior pressão antrópica nessas áreas e os efeitos negativos sobre a qualidade da água a ela associados. Além disso, as concentrações médias de fósforo descritas nessa pesquisa resultaram, em geral, maiores quando comparadas a levantamentos anteriores realizados no estado de São Paulo (e.g. LAMPARELLI, 2004);

- Do ponto de vista temporal, a análise sugeriu que não houve, entre 2005 e 2009, uma tendência de melhora da qualidade da água dos rios e reservatórios. As porcentagens de não conformidade apresentaram oscilações ao longo do período analisado, mas não se reduziram de forma significativa desde o início da vigência da Resolução CONAMA 357/2005. Assim, é necessário buscar mecanismos que possibilitem o estabelecimento de metas progressivas para recuperação da qualidade da água, por meio da inclusão dessas metas nos Planos de Recursos Hídricos, com prazos e estratégias que as tornem exequíveis;

- Sugere-se que seja resgatado o caráter de planejamento da Resolução CONAMA 357/2005, não limitando sua aplicação à utilização dos valores fixados para o simples acompanhamento da qualidade da água. O documento deve ser visto como um instrumento jurídico para o estabelecimento de metas progressivas para melhoria dos sistemas aquáticos em seus aspectos qualitativos, por meio dos comitês de bacia, com foco nos usos mais nobres a que a água se destina e com vistas à sustentabilidade em longo prazo. 


\section{Referências}

ACHLEITNER, S.; TOFFOL, S.; ENGELHARD, C.; RAUCH, W. (2005). The European Water Framework Directive: Water Quality Classification and Implications to Engineering Planning. Environmental Management, v. 35 , n. 4 , p. 517-525,

BELONDI, H.V. (2003). Enquadramento dos corpos d'água em classes de uso como instrumento de gestão ambiental e de recursos hídricos: estudo aplicado na bacia do rio Corumbataí - SP. Dissertação (Mestrado). Instituto de Geociências e Ciências Exatas, Universidade Estadual Paulista (UNESP). $161 \mathrm{p}$.

BRASIL. Resolução CONAMA 357, de 17 de março de 2005. Conselho Nacional de Meio Ambiente. Disponível em: <www.mma.gov.br/port/ conama/res/res05/res35705.pdf>. Acesso em: 8 out. 2010.

BONGARTZ, K.; STEELE, T.D.; BABOROWSKI, M.; LINDENSCHMIDT, K.E. (2007). Monitoring, assessment and modeling using water quality data in the Saale River Basin, Germany. Environmental Monitoring \& Assessment, v. 135, p. 227-240.

CALIJURI, M.C.; CUNHA, D.G.F.; POVINELLI, J. (2010). Sustentabilidade: um desafio na gestão dos recursos hídricos. São Carlos EESC-USP. 80 p.

CALIJURI, M.C.; CUNHA, D.G.F.; QUEIROZ, L.A.; MOCCELLIN, J.; MIWA, A.C.P. (2008). Nutrients and chlorophyll-a concentrations in tropical rivers of Ribeira de Iguape Basin, SP, Brazil. Acta Limnologica Brasiliensia, v. 20, n. 2, p. 131-138.

CARBALLO, R.; CANCELA, J.J.; IGLESIAS, G.; MARÍN, A.; NEIRA, X.X.; CUESTA, T.S. (2009). WFD indicators and definition of the ecological status of rivers. Water Resources Management, v. 23, p. 2231-2247.

CARONE, M.T.; SIMONIELLO, T.; MANFREDA, S.; CARICATO, G. (2009). Watershed influence on fluvial ecosystems: an integrated methodology for river water quality management. Environmental Monitoring and Assessment, v. 152, p. 327-342

CHEN, C.H.; LIU, W.L.; LEU, H.G. (2006). Sustainable Water Quality Management Framework and a Strategy Planning System for a River Basin. Environmental Management, v. 38, p. 952-973.

CETESB - Companhia Ambiental do Estado de São Paulo. Relatórios de Qualidade das Águas Superficiais no Estado de São Paulo dos anos de 2005, 2006, 2007, 2008 e 2009. Disponível em: <www.cetesb.sp.gov. br>. Acesso em: 20 out. 2010

CUNHA, D.G.F.; BOTTINO, F.; CALIJURI, M.C. (2010). Land use influence on eutrophication-related water variables: case study of tropical rivers with different degrees of anthropogenic interference. Acta Limnologica Brasiliensia, v. 22, n. 1, p. 35-45.

CUNHA, D.G.F. \& CALIJURI, M.C. (2010). Análise probabilística de ocorrência de incompatibilidade da qualidade da água com o enquadramento legal de sistemas aquáticos: estudo de caso do rio Pariquera-Açu (SP). Engenharia Sanitária e Ambiental, v. 15, n. 4, p. 337-346.

CUNHA, D.G.F.; DODDS, W.K.; CALIJURI, M.C. (2011). Defining nutrient and biochemical oxygen demand baselines for tropical rivers and streams in São Paulo State (Brazil): a comparison between reference and impacted sites. Environmental Management, v. 48, p. 945-956.

CUNHA, D.G.F.; OGURA, A.P.; CALIJURI, M.C. (2012). Nutrient reference concentrations and trophic state boundaries in subtropical reservoirs. Water Science and Technology, v. 65, p. 1461-1467.

DODDS, W.K.; BOUSKA, W.W.; EITZMANN, J.L.; PILGER, T.J.; PITTS, K.L.; RILEY, A.J.; SCHLOESSER, J.T.; THORNBRUGH, D.J. (2009). Eutrophication of U.S. freshwaters: analysis of potential economic damages. Environmental Science and Technology, v. 43, p. 12-19.

DODDS, W.K.; CARNEY, E.; ANGELO, R.T. (2006). Determining Ecoregional Reference Conditions for Nutrients, Secchi Depth and Chlorophyll a in Kansas Lakes and Reservoirs. Lake and Reservoir Management, v. 22, n. 2, p. 151-159.

DODDS, W.K. \& OAKES, R.M. (2004). A technique for establishing reference nutrient concentrations across watersheds affected by humans. Limnology and Oceanography: Methods, v. 2, p. 333-341.

DONADIO, N.M.M.; GALBIATTI, J.A.; DE PAULA, R.C. (2005). Qualidade da água de nascentes com diferentes usos do solo na bacia hidrográfica do córrego rico, São Paulo, Brasil. Engenharia Agrícola, v. 25, n. 1, p. 115-125.

FRANCO, R.A.M. \& HERNANDEZ, F.B.T. (2009). Qualidade da água para irrigação na microbacia do Coqueiro, Estado de São Paulo. Revista Brasileira de Engenharia Agrícola e Ambiental, v. 13, n. 6, p. 772-780

HENRY, R.; CARMO, C.F.; BICUDO, D.C. (2004). Trophic status of a Brazilian urban reservoir and prognosis about the recovery of water quality. Acta Limnologica Brasiliensia, v. 16, n. 3, p. 251-262.

IORISA, A.A.R.; HUNTERB, C.; WALKERC, S. (2008). The development and application of water management sustainability indicators in Brazil and Scotland. Journal of Environmental Management, v. 88, n. 4, p. 1190-1201.

LAMPARELLI, M.C. (2004). Graus de trofia de corpos d'água do Estado de São Paulo: avaliação dos métodos de monitoramento. Tese (Doutorado). Instituto de Biociências, Universidade de São Paulo (USP). 238p.

LEAL, A.C. Gestão das Águas no Pontal do Paranapanema - São Paulo. Tese (Doutorado). Instituto de Geociências, Universidade Estadual de Campinas (UNICAMP). 2000

MARCON, G. (2005). Avaliação da Política Estadual de Recursos Hídricos de São Paulo nas Bacias Hidrográficas dos rios Piracicaba, Capivari e Jundiaí. Tese (Doutorado). Faculdade de Saúde Pública, Universidade de São Paulo (USP). 274 p.

MARQUES, M.N.; COTRIM, M.B.; PIRES, M.A.F.; BELTRAME-FILHO, O. (2007). Avaliação do impacto da agricultura em áreas de proteção ambiental pertencentes à bacia hidrográfica do rio Ribeira de Iguape, São Paulo. Química Nova, v. 30, n. 5, p. 1171-1178.

MARTINELLI, L.A.; SILVA, A.M.; CAMARGO, P.B.; MORETTI, L.R.; TOMAZELLI, A.C.; SILVA, D.M.L.; FISCHER, E.G.; SONODA, K.C.; 
SALOMÃO, M.S.M.B. (2002). Levantamento das cargas orgânicas lançadas nos rios do estado de São Paulo. Biota Neotropica, v. 2, n. 2, p. 1-18.

MIDAGLIA, C.L.V. (2009). Proposta de implantação do Índice de abrangência Espacial de Monitoramento (IAEM) por meio da evolução da rede de qualidade das águas superficiais do estado de São Paulo. Tese (Doutorado). Universidade de São Paulo (USP). 229 p.

MOCCELLIN, J. (2006). A microbacia do rio Jacupiranguinha como unidade de estudo para a sustentabilidade dos recursos hídricos no Baixo Ribeira de lguape - SP. Dissertação (Mestrado). Escola de Engenharia de São Carlos, Universidade de São Paulo (USP). 151 p.

MOSCHINI-CARLOS, V.; BORTOLI, S.; PINTO, E.; NISHIMURA, P.Y.; FREITAS, L.G.; POMPÊO, M.L.M., DÖRR, F. (2009). Cyanobacteria and Cyanotoxin in the Billings Reservoir (São Paulo, SP, Brazil). Limnetica, v. 28, n. 2, p. 273-282

PIZELLA, D.G. \& SOUZA, M.P. (2007). Análise da sustentabilidade ambiental do sistema de classificação das águas doces superficiais brasileiras. Engenharia Sanitária e Ambiental, v. 12, n. 2, p. 139-148.

REIS, J.A.T. \& MENDONÇA, A.S.F. (2009). Análise técnica dos novos padrões brasileiros para amônia em efluentes e corpos d'água. Engenharia Sanitária e Ambiental, v. 14, n. 3, p. 353-362.
ROMÁN, R.M.S.; FOLEGATTI, M.V.; GONZÁLEZ, A.M.G.O. (2010). Water Resources Assessment at Piracicaba, Capivari and Jundiaí River Basins: A Dynamic Systems Approach. Water Resources Management, v. 24, p. 761-773.

SABIÁ, R.J. (2008). Estudo do padrão de emissão de poluentes para o enquadramento de rios intermitentes. Tese (Doutorado). Universidade Federal do Ceará (UFC).

SARDINHA, D.S.; CONCEIÇÃO, F.T.; SOUZA, A.D.G.; SILVEIRA, A.; JULIO, M.; GONÇALVES, J.C.S.I. (2008). Avaliação da qualidade da água e autodepuração do Ribeirão do Meio, Leme (SP). Engenharia Sanitária e Ambiental, v. 13, n. 3, p. 329-338.

SÃO PAULO. Decreto $n^{\circ}$ 10.755, de 22 de novembro de 1977. Dispõe sobre o enquadramento dos corpos de água receptores na classificação prevista no Decreto no 8.468, de 8 de setembro de 1976, e dá providências correlatas. Diário Oficial do Estado de São Paulo, 1977.

SEADE - Fundação Sistema Estadual de Análise de Dados. População e estatísticas vitais 2005, 2009 e 2010. Disponível em: <www.seade.gov. br>. Acesso em: 4 nov. 2010.

SILVA, S.C. \& RIBEIRO, M.M.R. (2007). Enquadramento dos corpos d'água e cobrança pelo uso da água na bacia o rio Pirama - PE. Engenharia Sanitária e Ambiental, v. 11, n. 4, p. 371-379. 